\title{
ON AN EXTENSION OF LOCALIZATION THEOREM AND GENERALIZED CONNER CONJECTURE
}

BY

\author{
SATYA DEO, TEJ BAHADUR SINGH AND RAM ANUGRAH SHUKLA
}

\begin{abstract}
Let $G$ be a compact Lie group. Then Borel-Segal-Quillen-Hsiang localization theorems are known for any $G$-space $X$ where $X$ is any compact Hausdorff space or a paracompact Hausdorff space of finite cohomology dimension. The Conner conjecture proved by Oliver and its various generalizations by Skjelbred are also known for only these two classes of spaces. In this paper we extend all of these results for the equivariant category of all finitistic $G$-spaces. For the case when $G=Z_{p}$ or $G=T$ (torus) some of these results were already proved by Bredon.
\end{abstract}

1. Introduction. The Borel-Segal-Quillen-Hsiang [10], [12] localization theorem states: Let $X$ be a paracompact (always assumed Hausdorff) space and $G$ a compact Lie group acting continuously on $X$ with finitely many orbit types. Let $X$ be of finite cohomological dimension over a coefficient ring $\Lambda$. Let $S \subset H^{*}\left(B_{G}, \Lambda\right)$ be a multiplicative system. Then the localized restriction homomorphism $S^{-1} H_{G}^{*}(X, \Lambda) \rightarrow$ $S^{-1} H_{G}^{*}\left(X^{S}, \Lambda\right)$ is an isomorphism. The Conner conjecture as proved by Oliver [11] states: Let $X$ be a paracompact space and $G$ a compact Lie group acting continuously on $X$ with finitely many orbit types. Let $\Lambda=Z, Z_{p}$ or $Q$ and assume that $X$ is of finite cohomology dimension over $\Lambda$. Then if $X$ is $\Lambda$-acyclic then so is $X / G$. Both of the above results hold when $X$ is compact Hausdorff even without the condition on number of orbit types and on the cohomology dimension of $X$. However, for paracompact spaces both of these conditions have been consistently and conveniently taken, not only in the above theorems and the results proved before them [7], but also in obtaining the generalized Conner conjecture as formulated and proved by Skjelbred [14]. In all these cases one can easily see that the condition on number of orbit types is needed. But the condition on the cohomology dimension of $X$ is not really needed except of course in the methods of proofs. Also it should be noted that the condition on the cohomology dimension of $X$ is not, in contrast to the condition on number of orbit types, a condition on the transformation group $(G, X)$. It depends on the coefficient ring. Naturally, therefore, one would like to substitute some condition on the transformation group, preferably a weaker one, in place of cohomology dimension of $X$ to achieve the same results. The objective of this paper is to prove all of the above-mentioned theorems by substituting the 'Swan' condition [4, p. 133], as has been already done by Bredon [4, Chapters III, VII] in proving several Smith-type theorems for $Z_{p}^{r}$-actions and $T^{r}$-actions. A

Received by the editors February 25, 1980.

1980 Mathematics Subject Classification. Primary 57S99; Secondary 55N30, 55M99. 
paracompact space satisfying the Swan condition is called finitistic. Bredon assumed that both $X$ and $X / T$ are finitistic. But now it has been proved [8] that when $X$ is finitistic and $G$ is a compact Lie group acting on $X$ then the orbit space $X / G$ is also finitistic. Since the class of finitistic spaces includes the class of compact Hausdorff spaces and the class of all paracompact spaces of finite covering dimension ( = cohomological dimension over $Z$ ), our results [Theorems 2.2, 3.3, 4.3, 4.4, and Corollary 4.5] not only extend the localization theorem, Conner conjecture and Skjelbred's generalized Conner conjecture, but also unify the methods of proofs given for the class of compact spaces and paracompact spaces of finite cohomological dimensions. We do not know whether or not a paracompact space of finite cohomological dimension over $Z$ is finitistic, yet our results are quite satisfactory and tend to support the point of view adopted by Bredon [4, Chapters III and VII] that the equivariant category of finitistic spaces is the appropriate category for the cohomological study of fixed point sets and orbit spaces. The localization theorem for finitisic spaces has been proved in $\$ 2$ and the Conner conjecture and generalized Conner conjecture for finitistic spaces have been done in $\S \S 3$ and 4.

2. Localization theorem. Let $G$ denote a compact Lie group and $\Lambda=Z_{p}$, or $Q$ be the ground ring for the cohomology to be used. In this section we shall work with sheaf cohomology with supports in the family of all closed sets. For paracompact spaces this cohomology agrees with the Čech cohomology with coefficients in arbitrary sheaf $\left[9\right.$, p. 228]. $H^{*}(X, \Lambda)$ will denote the cohomology of $X$ with coefficients in the constant sheaf $\Lambda$ and will be abbreviated as $H^{*}(X)$ unless explicitly mentioned otherwise. Let $E_{G} \rightarrow B_{G}$ denote the universal $G$-bundle and let $X \times E_{G}$ denote the $G$-space with diagonal action where $X$ is any paracompact $G$-space. The equivariant projection on the first factor induces the continuous map $\phi: X_{G} \rightarrow X / G$ with fiber over $x^{\prime} \in X / G$ homeomorphic to $B_{G_{x}}$. Here $G_{x}$ is the isotropy group of $x \in X, u: X \rightarrow X / G$ is the orbit map, and $u(x)=x^{\prime}$. The equivariant projection on the second factor induces the associated principal $G$ bundle $\pi: X_{G} \rightarrow B_{G}$ with $X$ as a fiber. Then $H^{*}\left(X_{G}\right)$, denoted by $H_{G}^{*}(X)$, is called the equivariant cohomology of the $G$-space $X$. Let $R=H^{*}\left(B_{G}\right)$ be the cohomology ring of the classifying space $B_{G}$ of $G$ and let $X$ be any $G$-space. Then $H_{G}^{*}(X)$ is an $R$-module via the map $\pi: X_{G} \rightarrow B_{G}$. Also the homomorphism $f^{*}: H_{G}^{*}(Y) \rightarrow H_{G}^{*}(X)$ induced by any equivariant map $f: X \rightarrow Y$ is an $R$-homomorphism (cf. [10, pp. 33, 34] for details). In particular, $\forall x^{\prime} \in X / G, H^{*}\left(E_{G} / G_{x}\right) \approx H^{*}\left(B_{G_{x}}\right)$, where $u(x)=$ $x^{\prime}$ is an $R$-module via the canonical quotient map $B_{G_{x}} \rightarrow B_{G}$. Now, let $S \subset R$ be any multiplicative system in $R$ and define

$$
X^{S}=\left\{x \in X \mid \text { no element of } S \text { is mapped to zero in } R \rightarrow H^{*}\left(B_{G_{x}}\right)\right\}
$$

Then one can easily prove

Proposition 2.1. Let $X$ be a $G$-space and $S$ any multiplicative system in $R=$ $H^{*}\left(B_{G}\right)$. Then:

(i) $X^{G} \subseteq X^{S} \subseteq X^{s} \forall s \in S$ and each of these subsets is a closed invariant subset of $X$ consisting of complete orbit types. 
(ii) If there is only a finite number of orbit types in $X$ then $X^{S}=X^{s}$ for some $s \in S$.

Since the inclusion map $X^{S} \subset X$ is equivariant, the restriction homomorphism $H_{G}^{*}(X) \rightarrow H_{G}^{*}\left(X^{S}\right)$ is an $R$-homomorphism. Therefore, the localized restriction $S^{-1} H_{G}^{*}(X) \rightarrow S^{-1} H_{G}^{*}\left(X^{S}\right)$ is also an $R$-homomorphism. The following is our localization:

THEOREM 2.2. Let $X$ be a finitistic space and $G$ a compact Lie group acting on $X$. Suppose $S$ is a multiplicative system in $R=H^{*}\left(B_{G}\right)$ and $s \in S$. Then the localized restriction $S^{-1} H_{G}^{*}(X) \rightarrow S^{-1} H_{G}^{*}\left(X^{s}\right)$ is an isomorphism. Furthermore, if the number of orbit types in $X$ is finite then the localized restriction homomorphism $S^{-1} H_{G}^{*}(X) \rightarrow$ $S^{-1} H_{G}^{*}\left(X^{S}\right)$ is also an isomorphism.

First of all we have

Proposition 2.3. Let $Y$ be a finitistic space and $\mathbb{Q}$ a sheaf of $\Lambda$-modules on $Y$. If $\alpha \in H^{i}(Y, \mathbb{Q})$ where $i>0$ then there is a positive integer $n_{0}$ such that $\alpha^{n}=0 \in$ $H^{*}(Y, Q), \forall n \geqslant n_{0}$.

Proof. Since $Y$ is a paracompact Hausdorff space the sheaf cohomology of $Y$ with coefficients in $Q$ is isomorphic to the Čech cohomology of $Y$ with coefficients in $\mathcal{Q}$, we find that $\forall k, H^{k}(Y, \mathcal{Q}) \approx \lim H^{k}(K(\mathcal{Q}), \mathcal{Q})$, where $K(\mathscr{U})$ denotes the nerve of the open cover $\mathcal{Q}$ of $Y$ and the limit is taken over the downward directed set of all open coverings of $Y$. Because $Y$ is finitistic, the set of all finite-dimensional open coverings of $Y$ forms a cofinal set. Hence we can assume that each $K(\mathscr{Q})$ is a finite-dimensional simplicial complex. Now suppose $\alpha$ is represented by some $\alpha^{\prime} \in H^{i}(K(\mathscr{U}), \mathscr{Q})$. If $K(\mathscr{U})$ is an $m$-dimensional simplicial complex then we choose $n_{0}$ so that $n_{0} \cdot i=k>m$ and then $0=\left(\alpha^{\prime}\right)^{n_{0}} \in H^{k}(K(\mathscr{Q}), \mathbb{Q})$ will represent $\alpha^{n_{0}}$ and so $\alpha^{n_{0}}=0$.

REMARK. Each element of positive degree in the cohomology algebra $H^{*}(Y, \mathbb{Q})$ of a finitistic space $Y$ is nilpotent. Of course, the sheaf $Q$ is understood to be with a given pairing.

The above remark is not valid if the space $X$ is not finitistic; for example, take $X$ to be the classifying space of a circle. Next let us consider a continuous map $f$ from a space $X$ to a finitistic space $Y$. Then the Leray spectral sequence of $f$, in which $E_{2}^{p q} \approx H^{p}\left(Y, \mathcal{F}^{q}(f)\right)$ where $\mathcal{H}^{q}(f)$ is the Leray sheaf of the map $f$, converges to $H^{p+q}(X)$. Suppose $F$ is the decreasing filtration of $H^{*}(X)$ such that $F^{p} / F^{p+1} \approx$ $E_{\infty}^{p q}$ and let $s \in F^{1}\left(H^{*}(X)\right) \subset H^{*}(X)$. Then we have the following

LEMMA 2.4. There exists a positive integer $n_{0}$ such that $s^{n_{0}}=0 \in H^{*}(X)$.

Proof. Without loss of generality we can assume that $s \in F^{1}\left(H^{n}(X)\right)$. For, $F^{1}\left(\bigoplus_{n>0} H^{n}(X)\right)=\bigoplus_{n>0} F^{1}\left(H^{n}(X)\right)$ means $s=s_{1}+s_{2}+\cdots+s_{r}$ where each $s_{i} \in F^{1}\left(H^{n_{i}}(X)\right)$ and therefore if a power of each $s_{i}$ vanishes in $H^{*}(X)$ then a suitable power of $s$ will also vanish in $H^{*}(X)$. Now since $\Lambda$ is a field we can assume that $s$ can be written uniquely as

$$
s=s_{1, n-1}+s_{2, n-2}+\cdots+s_{n, 0}
$$


where $s_{i, n-i} \in E_{\infty}^{i, n-i}, i=1, \ldots, n$. However, since $E_{\infty}^{i, n-i}$ is a subquotient of $E_{2}^{i, n-i} \approx H^{i}\left(Y, \mathcal{H}^{n-i}(f)\right)$ we can assume that $s_{i, n-i}$ is represented by $\alpha_{i, n-i} \in$ $H^{i}\left(Y, \mathcal{H}^{*}(f)\right)$ for each $i$. Recall that the cup product in the $E_{2}$ term agrees with the cup product in $H^{*}\left(Y, \mathcal{H}^{*}(f)\right)$ and the cup product in the $E_{\infty}$ term is induced by that of the $E_{2}$ term and is also compatible with the cup product in the cohomology of $H^{*}(X)$ (cf. [2, p. 143]). Since $Y$ is finitistic a power of $\alpha_{i, n-i}$ say $k_{i}$, vanishes in $H^{*}\left(Y, \mathcal{H}^{*}(f)\right)$. Therefore $\left(\alpha_{1, n-1}+\cdots+\alpha_{n \cdot 0}\right)^{k_{1} \cdots k_{n}}$ vanishes and this means $a$ suitable power of $s$ vanishes in $H^{*}(X)$.

Proof OF THEOREM 2.2. Let us consider the Leray spectral sequence of the map $\phi: X_{G} \rightarrow X / G$. Then the Leray spectral sequence of the map $\phi$ is such that $E_{2}^{p q} \approx H^{p}\left(X / G, \mathcal{F}^{q}(\phi)\right)$ and there is a decreasing filtration $F^{p}$ of $H^{*}\left(X_{G}\right)$ such that $F^{p} / F^{p+1} \approx E_{\infty}^{p q} \forall p, q \geqslant 0$. Also, it follows from the continuity [12, p. 552] of $H_{G}^{*}$-theory that the stalk of the Leray sheaf $\mathcal{F}^{q}(\phi)$ at the point $x^{\prime}=u(x) \in X / G$ is isomorphic to $H^{q}\left(B_{G_{x}}\right)$. First we prove our theorem for the case $X^{s}=\phi$. Since $X$ is finitistic $X / G$ is also finitistic. Now $X^{s}=\phi$ means $s$ is mapped to zero in $H^{*}\left(B_{G}\right) \rightarrow H^{*}\left(B_{G_{x}}\right) \forall x \in X$. Therefore $s$ is mapped to zero in each stalk of the Leray sheaf $\mathcal{H}^{*}(\phi)$. Hence $s$ is mapped to zero in $E_{2}^{0 *}=H^{0}\left(X / G, \mathcal{H}^{*}(\phi)\right)$ and consequently in $E_{\infty}^{0 *}$. Since the sequence

$$
0 \rightarrow F^{1}\left(H_{G}^{*}(X)\right) \rightarrow F^{0}\left(H_{G}^{*}(X)\right)=H^{*}\left(X_{G}\right) \rightarrow E_{\infty}^{0 *} \rightarrow 0
$$

is exact we find that $\pi^{*}(s) \in F^{1}\left(H_{G}^{*}(X)\right)$ where $\pi: X_{G} \rightarrow B_{G}$ is the bundle map. Since $X / G$ is finitistic, it follows from Lemma 2.4 that some positive power of $\pi^{*}(s)$ vanishes in $H^{*}\left(X_{G}\right)$, i.e., $S^{-1} H^{*}\left(X_{G}\right)=0$.

Now to prove the general case, it is sufficient in view of the exact sequence of the pair $\left(X, X^{s}\right)$ for $H_{G}^{*}$-theory, to show that $S^{-1} H_{G}^{*}\left(X, X^{s}\right)=0$. For this it is enough to show that for each $\alpha \in H_{G}^{*}\left(X, X^{s}\right)$ there is an $s^{\prime} \in S$ such that $s^{\prime} \cdot \alpha=0$. Now if $B_{G}^{k}$ denotes the $k$-skeleton of $B_{G}$ and $X_{G}^{k}=\pi^{-1}\left(B_{G}^{k}\right)$, then we know that the restriction homomorphism $H^{*}\left(X_{G}^{k+1}\right) \rightarrow H^{*}\left(X_{G}^{k}\right)$ is an isomorphism in degrees less than $k$ and, therefore, we may assume that

$$
\alpha \in H^{n}\left(X_{G},\left(X^{s}\right)_{G}\right)=H^{n}\left(X_{G}^{k},\left(X^{s}\right)_{G} \cap X_{G}^{k}\right) \text { for } k>n .
$$

Now since $X_{G}^{k}$ is paracompact Hausdorff and the nbds of $\left(X^{s}\right)_{G} \cap X_{G}^{k}$ in $X_{G}^{k}$ of the form $\left\{V_{G} \cap X_{G}^{k} \mid V\right.$ is an invariant nbd of $\left.X^{s}\right\}$ are cofinal, it follows from the continuity of $H_{G}^{*}$-cohomology $\left[12\right.$, p. 552] that there is an invariant nbd $V$ of $X^{s}$ such that $\alpha \in \operatorname{Im}\left(H_{G}^{*}(X, V) \rightarrow H_{G}^{*}\left(X, X^{s}\right)\right)$. On the other hand there is an invariant paracompact subspace $Y \subset X-X^{s}$ such that $X=V \cup$ Int $Y$. Since $Y^{s}=\phi$, some power, say $n$, of $\pi^{*}(s)$ vanishes in $H^{*}\left(Y_{G}\right)$. Once again it follows from the exact sequence of the pair $(X, Y)$ for $H_{G}^{*}$-theory that $\left(\pi^{*}(s)\right)^{n}$ is in

$$
\operatorname{Im}\left(H_{G}^{*}(X, Y) \rightarrow H_{G}^{*}(X)\right)
$$

and therefore is in $\operatorname{Im}\left(H_{G}^{*}(X\right.$, Int $\left.Y) \rightarrow H_{G}^{*}(X)\right)$. This means $\left(\pi^{*}(s)\right)^{n} \cdot \alpha$ lies in $\operatorname{Im}\left(H_{G}^{*}(X, V \cup \operatorname{Int} Y) \rightarrow H_{G}^{*}\left(X, X^{s}\right)\right)$. But, since $H_{G}^{*}(X, V \cup$ Int $Y)=0$, we find that $\left(\pi^{*}(s)\right)^{n} \cdot \alpha=0$.

The last part of the theorem follows from Proposition 2.1. Q.E.D.

Remark. A special case of our Theorem 2.2, viz., when $G=Z_{p}$ or $T$ and the coefficients are taken in the field $Z_{p}$ or $Q$, respectively, and when the multiplicative 
system $S$ was taken to be $S=\left\{1, t, t^{2}, \ldots, t^{n}, \ldots\right\}$ was observed by Bredon in [5, §2]. In that case $X^{S}=F$ (the fixed set) and $j^{*}: H^{*}\left(X_{G}\right) \rightarrow H^{*}\left(F_{G}\right)$ is an isomorphism in high degrees [4, p. 374] and, consequently, $\operatorname{Ker} j^{*}$ and Coker $j^{*}$ both vanish in high degrees. Therefore $S^{-1} R \otimes_{R} H_{G}^{*}(X) \rightarrow S^{-1} R \otimes_{R} H_{G}^{*}(F)$ is an isomorphism. Since localizing at $S$ is equivalent to tensoring by $S^{-1} R$ over $R$, we find that $S^{-1} H_{G}^{*}(X) \rightarrow S^{-1} H_{G}^{*}(F)$ is an isomorphism.

3. Conner conjecture for finitistic spaces. Our proof of the Conner conjecture for finitistic spaces will be partly based upon the following result, whose proof is given in [14].

THEOREM 3.1. Let $G$ be a connected simple Lie group and $X$ any paracompact $G$-space. Then there is a finite $G-C W$-complex $Z$ on which $G$ acts without fixed points and there is a spectral sequence with $E_{1}=C_{\text {cell }}^{*}\left(Z / G, \mathcal{H}^{*}\left(X / G_{c}\right)\right)$ converging to $H^{*}\left(S^{1}\right) \otimes H^{*}(X /(G))$. Here $c$ denotes a cell of $Z / G$, and for each $c, G_{c}$ is a proper closed subgroup of $G$. Similarly for reduced cohomology there is a spectral sequence $\tilde{E}_{1}=C_{\text {cell }}^{*}\left(Z / G, \tilde{\mathcal{H}} *\left(X / G_{c}\right)\right)$ converging to $H^{*}\left(S^{1}\right) \otimes \tilde{H}^{*}(X / G)$.

The coefficients in the above theorem are arbitrary. The proof of the theorem uses the existence of spheres of Floyd and Hsiang and the Conner conjecture for finite $G$-CW-complexes and the usual Borel construction for getting the spectral sequence. We shall also use the following (ibid.).

Proposition 3.2. Let $G$ be a compact Lie group acting semifreely on a paracompact space $X$ with fixed point set $F$. Then, with arbitrary coefficients, there is $a$ Mayer-Vietoris exact sequence

$$
\ldots \stackrel{\delta}{\rightarrow} H^{q}(X / G) \rightarrow H^{q}(F) \oplus H_{G}^{q}(X) \rightarrow H_{G}^{q}(F) \stackrel{\delta}{\rightarrow} \ldots
$$

Further, if $F \neq \phi$ then there is a reduced Mayer-Vietoris exact sequence

$$
\ldots \stackrel{\delta}{\rightarrow} \tilde{H}^{q}(X / G) \rightarrow \tilde{H}^{q}(F) \oplus \tilde{H}_{G}^{q}(X) \rightarrow \tilde{H}_{G}^{q}(F) \stackrel{\delta}{\rightarrow} \ldots,
$$

where $\tilde{H}_{G}^{*}$ denotes the reduced groups of the equivariant cohomology theory.

Now we can prove

THEOREM 3.3. Let $X$ be a finitistic space and $G$ a compact Lie group acting on $X$ with finitely many orbit types. Then, if $X$ is $\Lambda$-acyclic, so is $X / G$, where $\Lambda=Z_{p}, Z$ or $Q$.

Proof. It suffices to prove the theorem when $G$ is a finite group, $G=T$ acting semifreely or $G$ is a connected simple group. First suppose $G$ is finite. If $\Lambda=Z_{p}$ and $\tilde{H}^{*}\left(X, Z_{p}\right)=0$, then it follows from [4, Chapter III, Theorems 7.9, 7.11] that $\tilde{H}^{*}\left(X^{Z_{p}}, Z_{p}\right)=0$ and $\tilde{H}^{*}\left(X / Z_{p}, X^{Z_{p}}, Z_{p}\right)=0$. This means $\tilde{H}^{*}\left(X / Z_{p}, Z_{p}\right)=0$. Now by induction we find that $\tilde{H}^{*}\left(X / P, Z_{p}\right)=0$ for any $p$-Sylow subgroup $P$ of $G$. Now if $\mu^{*}: H^{i}\left(X / P, Z_{p}\right) \rightarrow H^{i}\left(X / G, Z_{p}\right)$ denotes the transfer and $\pi^{*}$ : $H^{i}\left(X / G, Z_{p}\right) \rightarrow H^{i}\left(X / P, Z_{p}\right)$ is the obvious homomorphism induced by projection, then $\mu^{*} \circ \pi^{*}$ is multiplication by $|G / P|$ which is relatively prime to $p$, and so this is an isomorphism. Since $\tilde{H}^{*}\left(X / P, Z_{p}\right)=0$ we find that $X / G$ is $Z_{p}$-acyclic. 
Next if $\Lambda=Z$ then $\tilde{H}^{*}\left(X, Z_{p}\right)=0$ for each prime $p$ and hence by the above result $\tilde{H}^{*}\left(X / G, Z_{p}\right)=0$. This means $\forall i H^{i}(X / G, Z)$ is divisible by any prime and consequently, by any integer $n$. But again because $\mu^{*} \circ \pi^{*}: H^{i}(X / G, Z) \rightarrow$ $H^{i}(X, Z) \rightarrow H^{i}(X / G, Z)$ is multiplication by $|G|$ we find that $\tilde{H}^{i}(X / G, Z)=0$ $\forall i$. Finally when $\Lambda=Q, H^{i}(X / G, Q) \approx\left(H^{i}(X, Q)\right)^{G}=0$ and the proof is complete for finite $G$.

Now let $G=T$ acting semifreely on $X$. The result follows from [4, Proposition 10.7 , p. 162] when $\Lambda=Z$. When $\Lambda$ is a field we take $S=\left\{1, t, t^{2}, \ldots, t^{n}, \ldots\right\} \subset$ $H^{*}\left(B_{G}, \Lambda\right)$ as our multiplicative system, then $X^{S}=F$ and the localization theorem with respect to $S$ implies that $F$ is also $\Lambda$-acyclic. In particular, $F \neq \phi$ and the reduced Mayer-Vietoris exact sequence implies that $\tilde{H}^{*}(X / G, \Lambda)=0$.

Finally suppose $G$ is connected and simple. In this case we apply the reduced spectral sequence of Theorem 3.1. By induction on $\operatorname{dim} G$ find that $\tilde{H}^{*}(X / G, \Lambda)=$ 0. Q.E.D.

REMARK. It is interesting to note that the methods of proofs given by Oliver [11] for the above result when $X$ is of finite cohomological dimension over $\Lambda$ do not extend to the case when $X$ is finitistic. However, his result for finite $\mathrm{CW}$-complexes has been obviously the basis for our extension for finitistic spaces.

4. Generalized Conner conjecture for finitistic spaces. In this section we generalize Skjelbred's results [14] for finitistic spaces. First we have the following result for $\Lambda=Z_{p}, Z$ or $Q$.

Proposition 4.1. Let $X$ be a finitistic space and $G=T$ act on $X$ semifreely. Let $Y$ be a closed invariant subset of $X$ and assume that $H^{i}(X, Y, \Lambda)=0$ for $i>n$. Then $H^{i}\left(X^{T}, Y^{T}, \Lambda\right)=0$ for $i>n$ and $H^{i}\left(X / G, Y / G \cup X^{T}, \Lambda\right)=0 \forall i>n$. A similar result is true for $G=Z_{p}$ with $\Lambda=Z_{p}$ also.

Proof. For $G=T$ the Smith-Gysin sequence [4, p. 162] is exact with arbitrary coefficients. Since $X$ is finitistic, $X / G$ is also finitistic and the proof now follows from Proposition 10.7 [ibid.]. For $G=Z_{p}$ and $\Lambda=Z_{p}$ the proof follows from Theorem 7.9 of $[4$, p. 144].

This gives

Proposition 4.2. Let $X$ be a finitistic space and $G=T$ act semifreely on $X$. Let $Y$ be a closed invariant subset of $X$ and let $F$ denote the fixed set. If $H^{i}(X, Y, \Lambda)=0$ for $i>n$ then the inclusion map $\left(F_{G}, F_{G} \cap Y_{G}\right) \rightarrow\left(X_{G}, Y_{G}\right)$ induces an isomorphism $H^{i}\left(X_{G}, Y_{G}, \Lambda\right) \rightarrow H^{i}\left(F_{G}, F_{G} \cap Y_{G}, \Lambda\right)$ for $i>n$. A similar result is true for $G=Z_{p}$ and $\Lambda=Z_{p}$.

Proof. Since $X$ is paracompact and the action of $T$ is semifree, we find that $\phi$ : $X_{G} \rightarrow X / G$ induces an isomorphism

$$
\phi^{*}: H^{i}(X / G, Y / G \cup F) \rightarrow H^{i}\left(X_{G}, Y_{G} \cup F_{G}\right)
$$

for arbitrary coefficients. Now the exact sequence of the triple $\left(X_{G}, Y_{G} \cup F_{G}, Y_{G}\right)$ and the above isomorphism give the long exact sequences

$$
\begin{aligned}
\ldots \rightarrow H^{k}(X / G, Y / G \cup F) & \rightarrow H^{k}\left(X_{G}, Y_{G}\right) \rightarrow H^{k}\left(F_{G}, F_{G} \cap Y_{G}\right) \\
& \rightarrow H^{k+1}(X / G, Y / G \cup F) \rightarrow \ldots
\end{aligned}
$$


for arbitrary coefficients. Now since $X$ is finitistic and $H^{i}(X, Y, \Lambda)=0$ for $i>n$, we find by Proposition 4.1 that $H^{k}\left(X_{G}, Y_{G}\right) \rightarrow H^{k}\left(F_{G}, F_{G} \cap Y_{G}\right)$ is an isomorphism for $k>n$.

Now we can prove

THEOREM 4.3. Let $G$ be a compact Lie group acting on a finitistic space $X$ with finitely many orbit types. Assume $Y$ is a closed invariant subset of $X$. Then, if $H^{*}(X, Y, \Lambda)$ is finitely generated, so is $H^{*}(X / G, Y / G, \Lambda)$.

Proof. First assume that $\Lambda$ is a prime field. It suffices to prove the theorem in case $G$ is a finite group of prime order, $G=T$ acting semifreely, or $G$ is connected simple. When $G=Z_{p}$ and Char $\Lambda=0$ or relatively prime to $p$, then $H^{*}(X / G, Y / G, \Lambda) \approx\left[H^{*}(X, Y, \Lambda)\right]^{G}$ and the theorem is trivially true. Thus we can assume that when $G=Z_{p}, \Lambda=Z_{p}$ and when $G=T, \Lambda$ is an arbitrary prime field. Then, by Proposition 4.2, the restriction homomorphism $H^{*}\left(X_{G}, Y_{G}, \Lambda\right) \rightarrow$ $H^{*}\left(F_{G}, F_{G} \cap Y_{G}, \Lambda\right)$ is an isomorphism in high degrees. Now consider the MayerVietoris exact sequence

$$
\begin{aligned}
\ldots \stackrel{\delta}{\rightarrow} H^{i}(X / G, Y / G, \Lambda) & \rightarrow H^{i}(F, F \cap Y, \Lambda) \oplus H_{G}^{i}(X, Y, \Lambda) \\
& \rightarrow H_{G}^{i}(F, F \cap Y, \Lambda) \stackrel{\delta}{\rightarrow} \ldots
\end{aligned}
$$

This implies that $H^{*}(X / G, Y / G, \Lambda) \rightarrow H^{*}(F, F \cap Y, \Lambda)$ has finite-dimensional (over $\Lambda$ ) kernel and cokernel. Since $\operatorname{dim}_{\Lambda} H^{*}(F, F \cap Y, \Lambda) \leqslant \operatorname{dim}_{\Lambda} H^{*}(X, Y, \Lambda)$ $<\infty$, it follows that $\operatorname{dim}_{\Lambda} H^{*}(X / G, Y / G, \Lambda)<\infty$. In case $G$ is connected simple we use the spectral sequence with $E_{1}=C_{\text {cell }}^{*}\left(Z / G, \mathcal{H}^{*}\left(X / G_{c}, Y / G_{c}, \Lambda\right)\right)$ converging to $H^{*}\left(S^{\prime}, \Lambda\right) \otimes H^{*}(X / G, Y / G, \Lambda)$. By induction on $\operatorname{dim} G$ we may assume that $\operatorname{dim}_{\Lambda} H^{*}\left(X / G_{c}, Y / G_{c}, \Lambda\right)<\infty$ for each cell $c$ of $Z / G$. Since $Z / G$ is a finite cell complex, it follows that $\operatorname{dim} E_{1}<\infty$ and, therefore, $\operatorname{dim}_{\Lambda} H^{*}(X / G, Y / G, \Lambda)$ $<\infty$.

Next assume that $\Lambda=Z$. If $G$ is a finite group, let $q:(X, Y) \rightarrow(X / G, Y / G)$ be the orbit map and $t: H^{*}(X, Y, Z) \rightarrow H^{*}(X / G, Y / G, Z)$ the transfer map. Then $t q^{*}: H^{*}(X / G, Y / G, Z) \rightarrow H^{*}(X / G, Y / G, Z)$ is multiplication by $m=|G|$, and hence Coker $t q^{*} \subset H^{*}\left(X / G, Y / G, Z_{m}\right)$. Since $t q^{*}$ factors through a finitely generated group it suffices to show that $H^{*}\left(X / G, Y / G, Z_{m}\right)$ is finitely generated. But that is immediate from the fact that $H^{*}\left(X / G, Y / G, Z_{p}\right)$ is finitely generated for each prime $p$. Having proved the theorem for finite $G$ and $\Lambda=Z$, we consider the case when $G=T$. Since the number of orbit types is finite we can assume the action of $T$ to be semifree, and the same argument as in the case of a field is valid in view of Proposition 4.2. Finally, to prove the theorem for general $G$ we assume the result for all $H$ with $\operatorname{dim} H<\operatorname{dim} G$. Thus we assume that $G$ is connected simple. Once again the spectral sequence $E_{1}=C_{\text {cell }}^{*}\left(Z / G, \mathcal{H}^{*}\left(X / G_{c}, Y / G_{c}, Z\right)\right.$ ), where $\operatorname{dim} G_{c}<\operatorname{dim} G$ converging to $H^{*}\left(S^{\prime}, Z\right) \otimes H^{*}(X / G, Y / G, Z)$, proves the result. Q.E.D.

Now a close examination of the proof of Theorem $2[14$, p. 631] reveals that in case $G$ is connected simple only the 'Conner conjecture' for finite $\mathrm{CW}$-complexes is used to prove that theorem for any paracompact $G$-space $X$. For $G=Z_{p}$ and $G=T$ acting semifreely, Proposition 4.2 justifies the same proof to be valid for 
finitistic spaces. Thus, nowhere in the proof, has the condition that $X$ is of finite cohomological dim over $k$, where $k=Z_{p}$ of $Q$, been used in an essential way. Hence we have also proved

THEOREM 4.4. Let $X$ be a finitistic space on which a compact Lie group $G$ is acting with finitely many orbit types. Let $\mathcal{F}$ be a monoid of equivariant self-maps of $X$. Then for each prime field $k$ (any characteristic) every simple subquotient of the $\mathcal{F}$-module $H^{*}(X / G, k)$ is a simple subquotient of $H^{*}(X, k)$. If $Y$ is a closed subset of $X$ invariant under $G$ and all of $f \in \mathcal{F}$ then each simple subquotient of the $\mathscr{F}$-module $H^{*}(X / G, Y / G, k)$ is a simple subquotient of the $\mathscr{F}$-module $H^{*}(X, Y, k)$.

This at once yields

COROllary 4.5. Let $X$ be a finitistic space on which a compact Lie group $G$ acts with finitely many orbit types and let $f$ be an equivariant self-map of $X$. Let $k$ be any prime field. Then each eigenvalue of the induced homomorphism on $H^{*}(X / G, k)$ is an eigenvalue of the induced homomorphism on $H^{*}(X, k)$ provided $\operatorname{dim}_{k} H^{*}(X, k)<\infty$.

\section{REFERENCES}

1. M. Atiyah and G. Segal, Equivariant $K$-theory, Lecture Notes, Univ. Warwick, 1965.

2. G. E. Bredon, Sheaf theory, McGraw-Hill, New York, 1967.

3. Cohomological aspects of transformation groups, Proc. Conf. Transformation Groups (New Orleans, 1967), Springer-Verlag, Berlin and New York, 1968, pp. 245-280.

4. __ Introduction to compact transformation groups, Academic Press, New York, 1972.

5. __ Fixed point set of actions on Poincaré duality spaces, Topology 12 (1973), 159-175.

6. A. Borel et al., Seminar on transformation groups, Ann. of Math. Studies, No. 46, Princeton Univ. Press, Princeton, N. J., 1960.

7. P. E. Conner, Retraction properties of orbit space of a compact transformation group, Duke Math. J. 27 (1960), 341-357.

8. S. Deo and H. S. Tripathi, Toral actions on finitistic spaces, Topology (to appear).

9. R. Godement, Théorie des faisceaux, Actualités Sci. Indust., Hermann, Paris, 1952.

10. Wu-Yi Hsiang, Cohomology theory of topological transformation groups, Springer-Verlag, Berlin and New York, 1975.

11. R. Oliver, A proof of the Conner conjecture, Ann. of Math. (2) 103 (1976), 637-644.

12. D. Quillen, The spectrum of an equivariant cohomology ring. I, Ann. of Math. (2) 94 (1971), 549-572.

13. T. Skjelbred, Acyclic orbit space and cohomology eigenvalues of equivariant maps, Preprint No. 8, Oslo, 1975.

14. $634-642$.

, Cohomology eigenvalues of equivariant mappings, Comment. Math. Helv. 53 (1978),

Department of MAThematics, University of JAMmU, JAMmU-180001, INDia

Department of Mathematics, University of Allahabad, Allahabad-211002, India 